\title{
Users' guide to Knowledge management with concept maps and mind maps: what are these and a proposal of a workflow
}

Arindam Basu

1 University of Canterbury

\begin{abstract}
In this article we describe concept maps, mind maps, and explain the differences between concept and mind maps. We also describe and their relative contributions to the process of assimilation and development of knowledge. We show with an example of motivation of students from a text to construct concept maps and mind maps and show how mind maps can take off from concept maps and reconstitute existing knowledge visually.
\end{abstract}

\section{Definitions}

Concept Maps

Defined by Arindam Basu

Mind maps

Defined by Arindam Basu

\section{Introduction}

Concept Maps refer to an interlinked structure of knowledge presentation where you can link concepts with each other using key phrases (referred to as linked words). The concepts linked with linked phrases constitute a proposition. Here are the rules of constructing concept maps based on the paper by Novak and Canas ${ }^{[1]}$. So the way it is going to work is like as follows:

1. Step 1: Start with a list of concepts. The concepts must be single words that are nouns or adjectives or descriptors of events or objects. If you are working on a reivew of air pollution and want to construct a concept map from which you want to develop a review, "air" and "pollution" will be your concepts. The concepts are put in circles or in squares

2. Step 2: Put these concepts on a "placeholder" in the canvas you want to work with. This "canvas" can be a digital canvas, or it can be a paper-pencil with sketches. The 
placeholder is referred to as "parking zone". The parking zone is temporary which will soon disappear or may hold additional concepts as the concept map grows.

3. Step 3: Decide the rank order of the concepts. Some concepts are higher order concepts that encapsulate many ideas that are subsumed under it, and there are other concepts that are less generic. For example, in the air pollution example, "pollution" would be an idea that is more generic, or "air" would be more generic and "criteria pollutants" that in turn refer to SOX, NOX, O3, PM10 are lower level concepts. The higher level concepts are going to be placed on top of the canvas, and the lower level concepts are going to be placed in the bottom of the canvas. The order is important and will depend on the context in which you will write.

4. Step 4: Once you have placed the concepts in an order that you decide, it is time for you to add connecting phrases or keywords that connect the different concepts. So, for the air pollution example, you might add a linked phrase "include" to link between "criteria pollutants" and "O3". Now you have a complete sentence "Criteria pollutants include O3" . This is referred to as a "proposition"

5. Step 5: Keep connecting the concepts using linked words and phrases.

Here is an example of a concept map on student motivation.

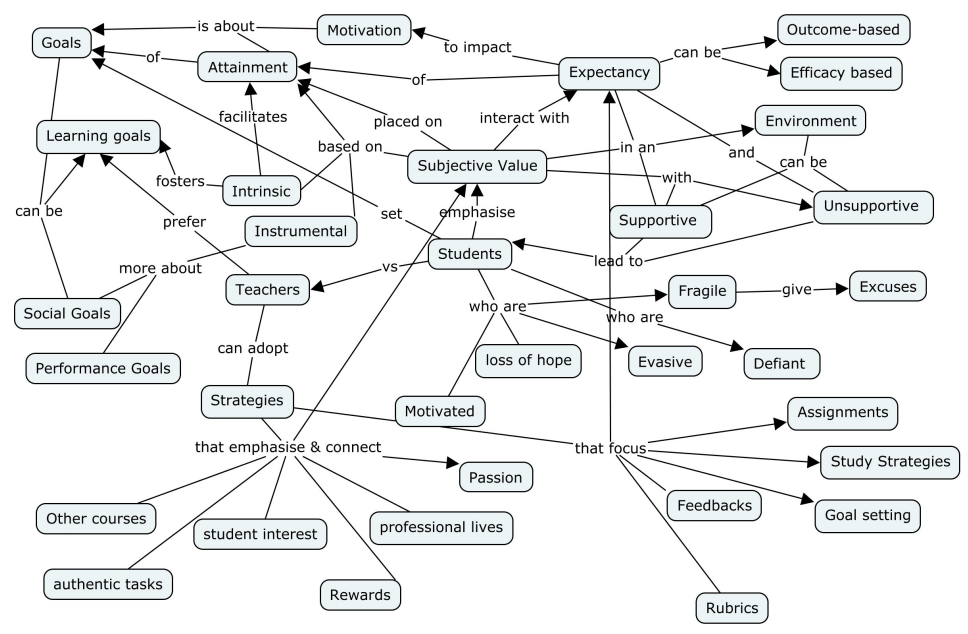

Figure 1. Concept map of student motivation. Note the concept words and the linking phrases connecting them.

In this map, for instance, you can see that:

- Higher order concepts such as "motivation", "goal", "expectancy", "value" are placed at the top

- Concrete expressions such as "authentic tasks", "rewards", "rubrics" are placed at the bottom of the canvas

- In general the arrows must point from a more generic to a more concrete concept 
using linking words that express an association. Hence linking words are ideally verbs or prepositions that indicate some action or some relationship.

Novak ${ }^{[1]}$ recommends two rules of constructing good concept maps:

1. The concepts must be organised hierarchically.

2. There must be appropriate linking phrases that will connect the hierarchically organised concepts.

With this in mind, linearly constructed concept maps, or concept maps that have many roots as nodes are not well designed. Also, concept maps that can connect each and every concept using linking phrases or linking words are better than concept maps that have sparse connections. Indeed, as Susan Ambrose ${ }^{[2]}$ writes in "How learning works", the difference between a novice and an expert learner is the number and density of the connections across the different concepts or knowledge units. Here, a proposition is a knowledge unit and you can form as many connections with named connectors as you can. Novak ${ }^{[1]}$ states for example that you can find new patterns or new ideas or missing ideas as you traverse the concept map trying to link different concepts. This might be important in writing for research projects and proposals where the challenge is to identify "gap". Proctor et.al. ${ }^{[3]}$ in their guide on writing research proposal for implementation science, for instance, has highlighted the role of finding a "gap". We propose that intuitively, you have best chance of finding such gaps in the assumptions and literature using a concept map. So, in summary, constructing a concept map is based on identifying the concepts first, then connecting the concepts using key phrases and rank ordering the concepts along a hierarchy. Besides these simple rules, it is important to be mindful to not use linear concept maps and disjo inted concepts with many root nodes.

\section{Can we construct concept maps for reading?}

Concept maps for reading can be constructed as you continue to read a paper or a book or book chapters. Ideally, such reading will consist of a number of different questions and so, a focused question is a first starting point for a concept map construction. You can connect the different focused questions to make up a coherent set of arguments. It is possible then to finally assemble a concept map that capsulates the questions that are embedded in the different parts of the concept map itself. So, one can construct concept maps for different questions that are answered in the different segments of a paper or a book and then reassemble a large concept map in the end. For example, the concept map shown above was constructed from Chapter 3 of the book by Susan Ambrose [2] where she discussed motivation for student learning and how to motivate student learners? What factors would motivate students to learn and how can teachers foster 
such processes. In this diagram we have taken a large overview of the processes, but it is equally possible to ask smaller questions and drill down deeper.

\section{Limitations of concept maps}

While concepts maps are excellent tools for visualising concepts and their relative positions, they are not usable for all situations. For example, Martin Eppler (2006) argues that concept maps are limited to situations where people who are not academically oriented to read complex information, or for describing procedural information are not well conveyed ${ }^{[4]}$. Therefore there are other visualisation tools that you can use to convey information, and here, moving from concept maps to mind maps might be useful.

How to use a mind map?

Concept maps give us a structure: start with higher order levels of concepts at the top and we work our way down to the more concrete instances to the bottom. Concept maps also provide a central question that we work with.

Mind maps were developed by T ony Buzan as a visual and rich visual representation of the mental processes, or ideas ${ }^{[4]}$. Mind maps start with a central topic and radiate outwards. This is not the starting point for concept maps where you can start anywhere and develop the concepts and connect them. You can use the concept maps to identify the high order concepts that then can feed the mind mapping process. Mind maps radiate from a central theme that you identified in the study phase or planning phase, and arranges the themes around a central circle where the ideas can then get developed further. With the back up of the inter-relationships of the concepts, the first and second order concepts are now presented in the mind map as radiating outwards. As an illustration, see the mind map associated or developed from the above concept map: 


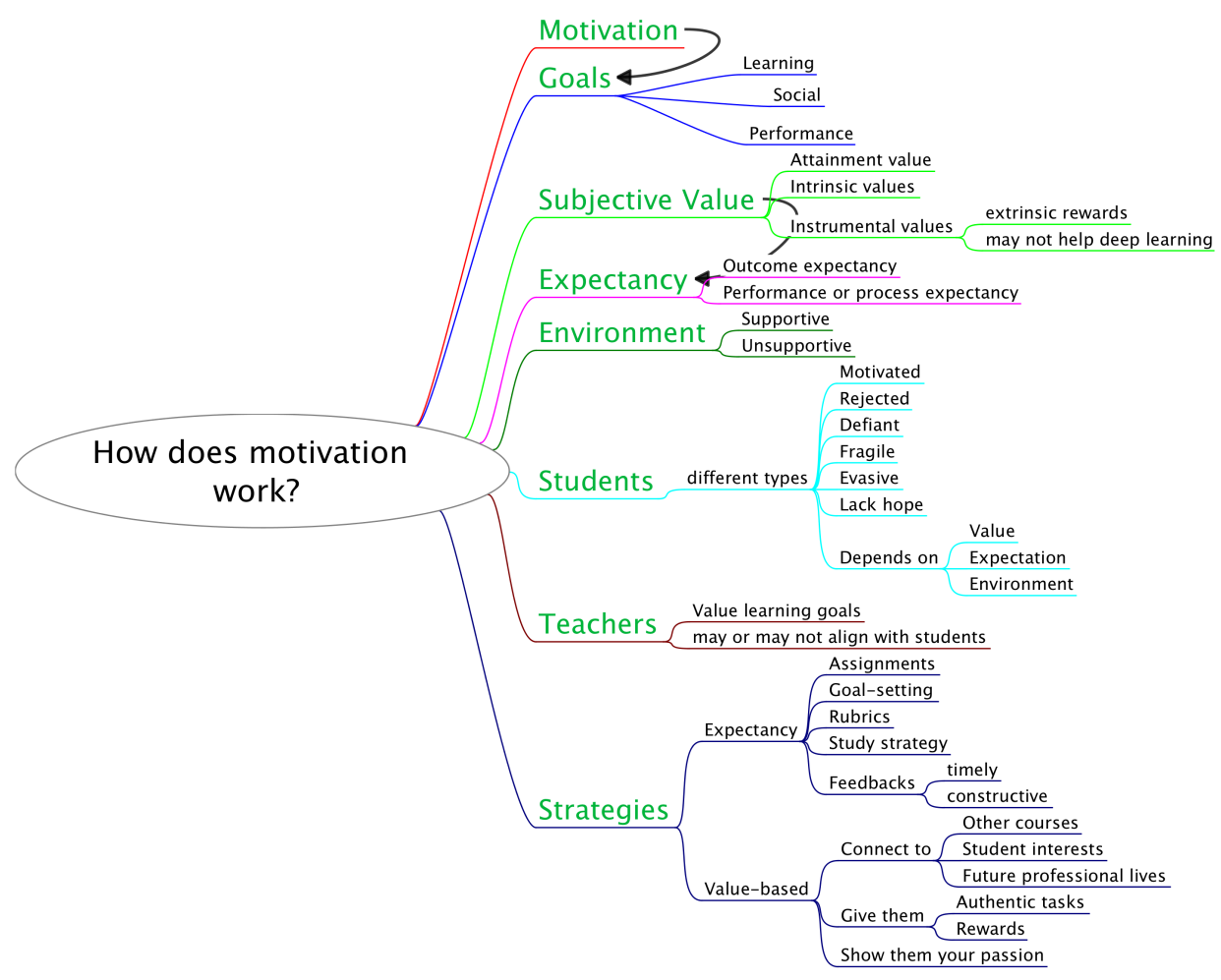

Figure 2. Development of the mind map from the concept map presented in the first figure. Note that the same concepts that were higher order are now radiating out from the parent topic. These could also be arranged differently

\section{An intuitive workflow integrating mind maps and concept maps}

In developing a knowledge base, we start with ideating and reading. The many reading of the journal articles, books, monographs can be consolidated using one or more concept maps in the first phase. In subsequent phases, the concept maps could be redrawn to consolidate the key concepts and find linkages between them or identify gaps in the key concepts that can be addressed using future research or more studying. When transferring concept maps to written or presentation format, it is intuitive to construct mind maps that would then be able to allow us add information, analyses, data, tables, figures to the concepts and embellish them further as we can develop statements out of the mind maps. In order to construct mind maps from concept maps, we identify the core question, the first order headings from the question that would present as top level hierarchy in the concept maps and then radiate out. Where concept maps move from top down with concepts and linked phrases, the mind maps move from centre to outwards with subconcepts and ideas that then get added or edited. The final products could be data analyses, or free text with ideas derived and made storyboards out of mind 
maps that originated in the concept maps.

\section{References}

1. a, b, c https://cmap.ihmc.us/docs/theory-of-concept-maps

2. a, b Susan A. Ambrose, Michae/W. Bridges, Michele DiPietro, Marsha C. Lovett, Marie K. Norman. How Learning Works.

3. ^ Enola K Proctor, Byron J Powell, Ana A Baumann, Ashley M Hamilton, Ryan L Santens. (2012). Writing implementation research grant proposals: ten key ingredients. Implementation Sci, vol. 7 (1)

4. a,b Martin J Eppler. (2006). A Comparison between Concept Maps, Mind Maps, Conceptual Diagrams, and Visual Metaphors as Complementary Tools for Knowledge Construction and Sharing. Information Visualization, vol. 5 (3), 202-210. 